\title{
E.S.C.L.O.S.A.: programa de orientación como ayuda al alumnado universitario en el EEES
}

\author{
E.S.C.L.O.S.A.: a guidance programme as help for College Freshmen in the EEES \\ Carmen $\mathrm{M}^{\mathrm{a}}$ Hernández Garre*, Borja Avilés Soler* \\ *Departamento de Educación, Universidad de Almería, España
}

\begin{abstract}
Resumen
En el Espacio Europeo de Educación Superior (EEES), como sistema educativo universitario actual, el aprendizaje se concibe como un proceso de construcción de significado, en el que el alumnado construye sus propios conocimientos, adoptando un papel mucho más activo, autónomo, autorregulado y al mismo tiempo conocedor de sus propios procesos cognitivos. El trabajo que se propone recoge la eficacia de un programa de intervención psicopedagógica que atiende a las necesidades en aspectos tanto formativos y vocacionales como socioafectivos de los estudiantes universitario de nuevo ingreso de la Facultad de Ciencias de la Educación de la Universidad de Granada.
\end{abstract}

\section{Abstract}

In The European Higher Education Area, as the current university educational system, the learning process is understood as a process of meaning construction, where the student constructs his own knowledge, adopting a much more active role, independent, self-directed and, at the same time, aware of his own cognitive process. The task proposed, involves the efficiency of a programme of psycho-pedagogical intervention that attends to the necessities of the university students of the University of Granada in different aspects such a as: formative, vocational as well as social affective.

Palabras clave: orientación, alumnado universitario, adaptación académica, programa universitario.

Keywords: guidance, college freshmen, academic adjustment, college programs.

\section{Método}

\section{Participantes}

A través de un muestreo aleatorio simple entre los estudiantes de primero de la titulación de Grado de Maestro en Educación Infantil, se selecciona a una muestra de 50 de los que finalmente participan 40 . Estos a su vez se dividieron de forma aleatoria en 20 estudiantes para el grupo control y 20 estudiantes para el grupo experimental por lo que el programa E.S.C.L.O.S.A., se realizó con el grupo experimental. De tal modo que el programa se implementó en 20 estudiantes pertenecientes a dicha titulación, con edades comprendidas entre los 18 y 20 años.

Tabla 1.

Distribución de la muestra

\begin{tabular}{lcc}
\hline & Frecuencia & $\begin{array}{c}\text { Porcentaje } \\
\text { representatividad (\%) }\end{array}$ \\
\hline Grupo Control & 20 & $13.9 \%$ \\
Grupo Experimental & 20 & $13.9 \%$ \\
$\quad$ Subtotal & 40 & $28 \%$ \\
\hline $\begin{array}{l}\text { Total (mortalidad } \\
\text { muestral) }\end{array}$ & 4.567 & 4.567 \\
\hline \multicolumn{2}{c}{ * Mortalidad muestral de 5 sujetos en el Grupo Control }
\end{tabular}

* Mortalidad muestral de 5 sujetos en el Grupo Contro

\section{Instrumentos}

Los instrumentos utilizados para la recogida de información sobre el alumnado fueron los siguientes:

- Variables Sociodemográficas: Cuestionario elaborado ad hoc., compuesto por 42 ítems agrupados en 5 subescalas que recogen datos sobre las características sociales y demográficas de los estudiantes.

- Comprensión lectora y ortografía: Para evaluar el área instrumental de lectura se utiliza un instrumento elaborado ad hoc (González, 2007), siguiendo las recomendaciones para la elaboración de pruebas de comprensión lectora interpretativa (Gómez-Villalba, Pérez, González, Hoyos y Cañado, 2000) y que incluye: prueba de comprensión lectora y vocabulario. El área de ortografía se evalúa utilizando las Pruebas para el diagnóstico cualitativo de la ortografía en el ciclo III de la Educación Primaria (Pérez, Galera y Pérez, 1998), en sus dos variantes: prueba de dictado y prueba de uso del plural, signos de acentuación y de puntuación.

- Hábitos de estudio: Se aplicó el Inventario de Hábitos de Estudio (I.H.E.) de Pózar, (2002).

- Habilidades sociales: Se utilizó la Escala de Habilidades Sociales (E.H.S.) (Gismero, 2002), compuesto por 33 ítems agrupados en 6 factores.

- Autoconcepto: El instrumento de evaluación empleado fue el Test de Autoconcepto Forma 5 (AF5), de García y Musitu (2001), integrado por 36 ítems. 


\section{Procedimiento}

El estudio toma como referencia el modelo de evaluación que proponen Stufflebeam y Shinkfield (1993), comúnmente conocido por las siglas CIPP, es decir, Contexto, Entrada (Input), Proceso y Producto.

- Evaluación del contexto: se estableció un análisis de necesidades para tener un conocimiento, lo más exhaustivo posible de las características sociodemográficas, académicas y sociopersonales del alumnado universitario de nuevo ingreso en las titulaciones de Maestro en Educación Infantil.

- Evaluación de entrada: Para dar respuesta a las necesidades detectadas en el alumnado se procede a diseñar un programa de intervención psicopedagógica. Para ello se determinan los recursos necesarios y se establecen los recursos humanos y materiales adecuados para llevar a cabo el programa al que denominamos E.S.C.L.O.S.A., cuyas siglas hacen referencia a: Estilos Socioculturales, Comprensión Lectora, Ortografía, Sociales y Autoconcepto.

- Evaluación del proceso: En esta fase, se pone en marcha el programa de intervención psicopedagógica E.S.C.L.O.S.A. El programa se desarrolla a través de cuatro talleres diferenciados: Taller de comprensión lectora, Taller de ortografía, Taller de hábitos, técnicas de estudio y autorregulación y Taller de habilidades sociales. El programa en su conjunto tiene una fase presencial de 32 horas y un número de sesiones no presenciales (16 horas), de tal manera, que finalmente las sesiones presenciales de los diferentes talleres se desarrollan durante 8 semanas, con una duración de 4 horas semanales, quedando organizadas del siguiente modo (Véanse Figuras 1, 2, 3 y 4).

\begin{tabular}{|c|c|c|c|}
\hline & CONTENIDOS & OBJETIVOS & DURACIÓN \\
\hline SESIÓN 1 & $\begin{array}{l}\text { La comprensión } \\
\text { lectora. La eficacia } \\
\text { lectora }\end{array}$ & $\begin{array}{l}\text { - Tomar conciencia de } \\
\text { los distintos procesos } \\
\text { implicadosen la lectura. }\end{array}$ & 2 horas \\
\hline SESIÖN 2 & $\begin{array}{l}\text { Tecnicas para la } \\
\text { comprension. El } \\
\text { resumen y ei } \\
\text { subrayado }\end{array}$ & $\begin{array}{l}\text { - Conocer y saber ufilizar } \\
\text { las estrategias mas } \\
\text { eficoces para facilitarlo } \\
\text { comprensión lectora. }\end{array}$ & 2 horas \\
\hline SESIÓN 3 & $\begin{array}{l}\text { Tésnicas para la } \\
\text { comprensión. El } \\
\text { esquema y el mapa } \\
\text { conceplual }\end{array}$ & $\begin{array}{l}\text { Conocer y saber utilizar } \\
\text { las estrategias más } \\
\text { eficaces para facilitar lo } \\
\text { comprensión lectoro }\end{array}$ & 2 horas \\
\hline SESIÓN 4 & $\begin{array}{l}\text { La composición de } \\
\text { textos: la redaceión }\end{array}$ & $\begin{array}{l}\text { - Saber el proce dimiento } \\
\text { correcto en la } \\
\text { elaboración de textos. }\end{array}$ & 2 horas \\
\hline
\end{tabular}

Figura 1. Taller de comprensión lectora

\begin{tabular}{|c|c|c|c|}
\hline & CONTENIDOS & OBJETIVOS & DURACIÓN \\
\hline SESIÓN 1 & $\begin{array}{l}\text { Ortografia. Normas } \\
\text { básicas. }\end{array}$ & $\begin{array}{l}\text { - Tomar conciencia de las } \\
\text { distintas normas } \\
\text { ortográficas. }\end{array}$ & 2 horas \\
\hline SESIÓN 2 & Vocabulario básico. & $\begin{array}{l}\text { - Conocer y saber utilizar } \\
\text { las normas básicas de } \\
\text { ortografía. }\end{array}$ & 2 horas \\
\hline SESIÓN 3 & $\begin{array}{l}\text { Acentuación de } \\
\text { formas verbales con } \\
\text { pronombres } \\
\text { encliticos. }\end{array}$ & $\begin{array}{l}\text { - Conocer las reglas } \\
\text { ortográficas de } \\
\text { determinadas formas } \\
\text { verbales. }\end{array}$ & 2 horas \\
\hline SESIÓN 4 & $\begin{array}{l}\text { Signos de } \\
\text { puntuación. }\end{array}$ & $\begin{array}{l}\text { - Saber situar los signos de } \\
\text { puntuación en un texto. }\end{array}$ & 2 horas \\
\hline
\end{tabular}

Figura 2.Taller de ortografía

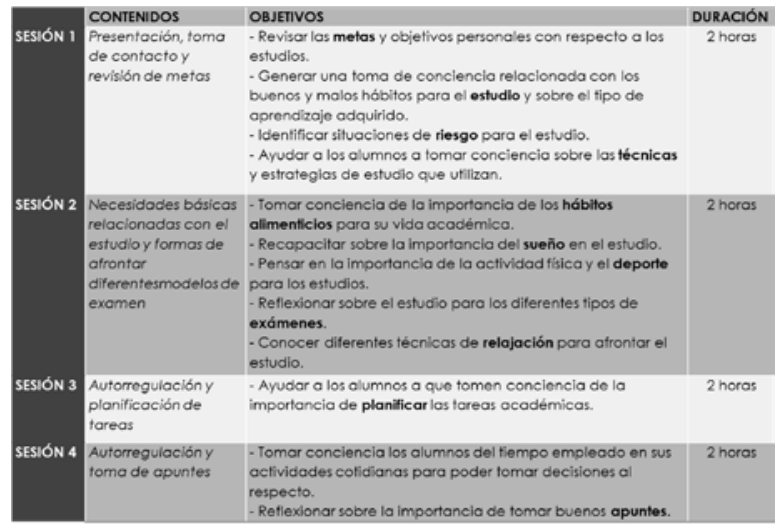

Figura 3. Taller de Hábitos, Técnicas de Estudio y Autorregulación

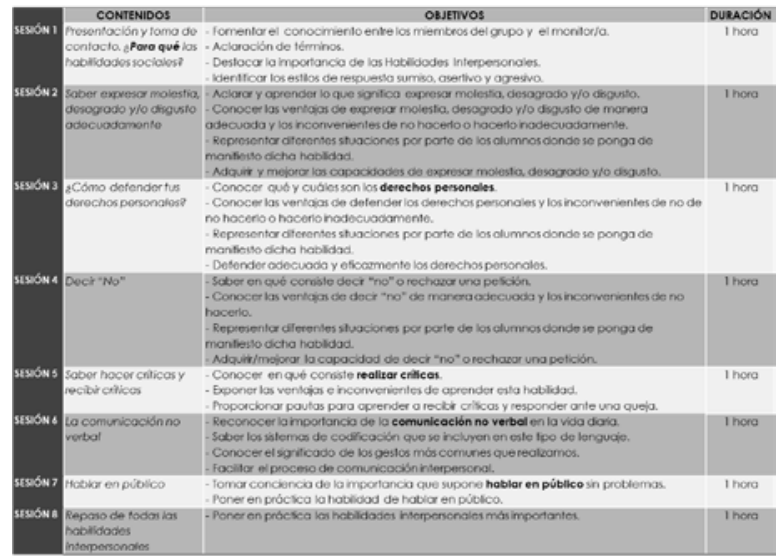

Figura 4. Taller de habilidades sociales

- Evaluación del producto: En esta fase se evaluaron las variables establecidas tras la finalización de las sesiones presenciales del programa. Una vez concluido este proceso, se concreta un día para su entrega y posteriormente se les cita individualmente para proporcionarles feedback sobre sus ejercicios y corregir los posibles errores.

Por otro lado, para comprobar la eficacia del programa E.S.C.L.O.S.A. se obtuvieron medidas pre- y post-tratamiento de ambos grupos, pero solamente el grupo experimental recibió el tratamiento o programa de intervención.

\section{Resultados}

Se presentan a continuación los resultados obtenidos en la evaluación de las diferentes variables contempladas en el programa de intervención E.S.C.L.O.S.A.

- Los resultados de comprensión lectora y ortografía, una vez realizada la prueba Chi-cuadrado al tratarse de un material elaborado ad hoc y de datos nominales, se pudieron constatar diferencias significativas en todas las dimensiones de las pruebas (significado de las palabras, comprensión del texto, ortografía dictado de palabras y ortografía plural, etc.) excepto en estrategia utilizada para el significado de las palabras, 
(Tabla 2) ya que el alumnado utilizó estrategias similares para definir el significado de las palabras extraídas del texto.

Tabla 2.

Chi-Cuadrado Pretest y Postest. Comprensión lectora y ortografía

\begin{tabular}{|c|c|c|}
\hline & \multicolumn{2}{|c|}{$\begin{array}{l}\text { Chi-Cuadrado } \\
\mathrm{p}<.05\end{array}$} \\
\hline & $\begin{array}{c}\text { PRETEST } \\
\text { Control } \\
\text { Experimental }\end{array}$ & $\begin{array}{c}\text { POSTEST } \\
\text { Control } \\
\text { Experimental }\end{array}$ \\
\hline Significado de las palabras & .10 & .00 \\
\hline $\begin{array}{l}\text { Estrategia utilizada para el sig. } \\
\text { de las palabras }\end{array}$ & .12 & .06 \\
\hline Comprensión del texto & .00 & .00 \\
\hline $\begin{array}{l}\text { Ortografía. Dictado de } \\
\text { palabras }\end{array}$ & .00 & .02 \\
\hline $\begin{array}{l}\text { Ortografía: plural, completa } \\
\text { frases, pon tildes, sig. } \\
\text { puntuación, completa frases }\end{array}$ & .00 & .00 \\
\hline
\end{tabular}

- Los resultados obtenidos en la variable hábitos de estudio, una vez realizada un ANOVA mostraban diferencias significativas en 4 de sus 5 dimensiones (Tabla 3).

Tabla 3.

ANOVA Pretest y Postest. Hábitos de estudio

\begin{tabular}{lcc}
\hline & \multicolumn{2}{c}{ ANOVA } \\
& $\begin{array}{c}\text { PRETEST } \\
\text { Control } \\
\text { Experimental }\end{array}$ & $\begin{array}{c}\text { POSTEST } \\
\text { Control } \\
\text { Experimental }\end{array}$ \\
\hline Ambiente & .30 & .00 \\
Planificación & .28 & .00 \\
Materiales & .01 & .06 \\
Asimilación & .01 & .00 \\
Sinceridad & .65 & .00 \\
\hline
\end{tabular}

- En cuanto a las habilidades sociales de los estudiantes, se hallaron diferencias significativas en la medida postest en todas sus dimensiones (Tabla 4) excepto en la dimensión iniciar interacciones positivas con el sexto opuesto pudiendo ser debido a que no se trabajó dicha dimensión en el programa.

Tabla 4.

ANOVA Pretest y Postest. Habilidades sociales

\begin{tabular}{ccc}
\hline \multicolumn{2}{c}{ ANOVA } \\
$\mathrm{p}<.05$ \\
\hline PRETEST & POSTEST \\
& Control & Control \\
Experimental & Experimental \\
\hline
\end{tabular}

Autoexpresión en situaciones

sociales

Defensa de los propios

derechos como consumidor

Expresión de enfado o

disconformidad

Decir No y cortar interacciones

Hacer peticiones
Iniciar interacciones positivas

con el sexo opuesto

.17 .17

- Por último, en la evaluación del autoconcepto de los estudiantes, tras la realización de una ANOVA se apreciaban diferencias significativas en la medida postest, en cada una de sus dimensiones (Tabla 5).

Tabla 5.

ANOVA Pretest y Postest. Autoconcepto

\begin{tabular}{lcc}
\hline & \multicolumn{2}{c}{ ANOVA } \\
& \multicolumn{2}{c}{ PRETEST } \\
& $\begin{array}{c}\text { Control } \\
\text { Experimental }\end{array}$ & $\begin{array}{c}\text { POSTEST } \\
\text { Control } \\
\text { Experimental }\end{array}$ \\
\hline Académico/laboral & .84 & .00 \\
Social & .14 & .00 \\
Emocional & .95 & .00 \\
Familiar & .17 & .00 \\
Físico & .09 & .00 \\
\hline
\end{tabular}

\section{Discusión}

El alumnado universitario de nuevo ingreso presenta una serie de necesidades a las que la institución universitaria debiera hacer frente. Los estudiantes universitarios noveles requieren se atendidos de manera integral en competencias tanto académicas como en estrategias de tipo socioafectivo. Por tanto, se hace evidente la necesidad de proporcionarles acciones que permitan conocer sus dificultades reales en todos estos aspectos.

Con la elaboración e implementación del programa E.S.C.L.O.S.A. el alumnado participante obtuvo un menor número de errores ortográficos y de comprensión lectora tras el entrenamiento en dichas áreas, asimismo mejoraron las condiciones ambientales en que se desarrollaba su estudio y tenían una planificación más adecuada del mismo. De igual modo, mejoraron sus habilidades sociales tras la participación en el programa. Aunque no se intervino directamente en la variable autoconcepto, la mejoría en las demás dimensiones entrenadas influyó positivamente en el autoconcpeto del alumnado participante.

Los resultados obtenidos permiten afirmar que los estudiantes universitarios noveles que reciben una formación acorde con sus necesidades podrán mejorar su adaptación a la institución y tendrán más posibilidades de permanecer en ella, así como tener éxito en sus estudios si disponen del acceso a programas como el diseñado. No obstante, es necesario señalar la conveniencia de replicaciones posteriores con una muestras más amplia de estudiantes.

\section{Referencias}

García, F. y Musitu, G. (1999). AF5. Autoconcepto Forma 5. Madrid: TEA. 
Gismero, E. (2000). EHS Escala de Habilidades Sociales. Madrid: TEA.

Gómez-Villalba, E., Pérez J., González, C., Hoyos. C. y Cañado. M. (2000). Desarrollo de las capacidades de los alumnos de Educación Primaria en el área de lengua castellana y literatura. Granada: Grupo Editorial Universitario.

González, C. (2007). Instrumento elaborado ad hoc.
Pérez, J., Galera, R., y Pérez, M. L. (1998). Pruebas para el diagnóstico cualitativo de la ortografía en el ciclo III de la Educación Primaria. Valladolid: La Calesa.

Pózar, F. (2002). IHE. Inventario de hábitos de estudio. Madrid: TEA.

Stufflebeam, D. L. y Shinkfield, A. J. (1993). Evaluación sistemática. Guía teórica y práctica. Madrid: Paidós-MECUGR. 